\title{
A SAMPLER OF DIFFRACTION AND REFRACTION OPTICALLY VARIABLE IMAGE ELEMENTS
}

\author{
${ }^{1 *}$ Miroslav HORÁČEK, ${ }^{1}$ Stanislav KRÁTKÝ, ${ }^{1}$ Milan MATĚJKA, ${ }^{1}$ Jana CHLUMSKÁ, \\ ${ }^{1}$ Petr MELUZíN, ${ }^{2}$ Jiří PIRUNČíK, ${ }^{2}$ Ivo AUBRECHT, ${ }^{2}$ Marek KOTRLÝ, ${ }^{1}$ Vladimír KOLAŘíK \\ ${ }^{1}$ Institute of Scientific Instruments of the CAS, v. v. i., Brno, Czech Republic, EU, " $\underline{\text { mih@isibrno.cz }}$ \\ ${ }^{2}$ Institute of Criminalistics of the Police of the Czech Republic, v. v. i., Prague, Czech Republic, EU
}

https://doi.org/10.37904/nanocon.2021.4371

\begin{abstract}
Diffraction and refraction optically variable image elements are basic building blocks of planar structures for advanced security of documents and valuables. A sampler formed by an array of 36 diffraction structures binary, tertiary, quaternary and blazed gratings (period range $400 \mathrm{~nm}-20,000 \mathrm{~nm}$ ) - represents a cross-section throughout technological steps - mastering, galvanic replication and embossing. Electron-beam writing technology with Gaussian beam and electron energy of $100 \mathrm{keV}$, with very small forward scattering of high energy electrons and with the possibilities to create a linear grating with the minimal period of $100 \mathrm{~nm}$, was used to create the master. An important advantage of high-resolution electron-beam lithography is its substantial flexibility in combining possible planar structures with significantly different parameters, such as very dense and relatively shallow structures together with deep structures (approx. 10 microns) with precise shapes (micro-lenses or Fresnel structures). For protection of documents and valuables, interesting results are induced with planar optical structures consisting of non-periodic arrangements, which are characterized by high robustness to counterfeiting and imitation.

While the origination process is available for grating period down to $100 \mathrm{~nm}$, the mass replication technology appears to be a bottleneck of the entire technological process. Measurement of topology and profiles of the structures by atomic forces microscope and documenting the quality of technological process of the three steps of replication of planar optically variable elements was performed for all 36 structure types of sampler.
\end{abstract}

Keywords: Diffraction; refraction; optically variable image element; e-beam writer; mastering; galvanic replication; embossing; security; valuables

\section{INTRODUCTION}

Various sophisticated techniques are involved in protection of documents and valuables against counterfeiting or tampering and the most wide-spread non-printing types are the ones that rely on spectral and directional redistribution of incident white light by surface-relief structures embossed in a foil. These structures are commonly denoted as variable image elements and may be characterized with varying appearance that depends on geometry of illumination and observation or even on polarization state.

While the electron-beam writing origination process is available for grating period down to $100 \mathrm{~nm}$, the mass replication technology appears to be a bottleneck of the entire technological process - mastering, galvanic replication and embossing. Problems of individual technological steps of the production process are demonstrated on a sampler of 36 mesh diffraction structures - binary $(2 \mathrm{~L})$, tertiary $(3 \mathrm{~L})$, quaternary $(4 \mathrm{~L})$ (with range of periods from $400 \mathrm{~nm}$ to $4,320 \mathrm{~nm}$ ) and blazed gratings (16L) (with range of periods from 2,000 $\mathrm{nm}$ to 20,000 nm), see Figure 1. 


\section{MASTERING}

Electron-beam lithography (EBL) is recognized to be a versatile origination tool as regards the possibility of creating very fine relief features and a general design. Electron-beam writer with Gaussian beam and electron energy of $100 \mathrm{keV}$ was used to create the master. With very small forward scattering of high energy electrons, it is possible to create a linear grating with a period of down to $100 \mathrm{~nm}$. Advantage of this versatility consist in combining possible planar structures with significantly different parameters: Very dense though relatively shallow structures may be combined together with deep structures (approx. 10 microns) with precise shapes. Micro lenses, Fresnel structures and non-periodic arrangements may serve as examples of the latter. Hence, EBL induced interesting results for protection of documents and valuables, which are characterized by high robustness to counterfeiting and imitation.

With the EBL the sampler was designed as an array of $6 \times 6$ structures and realized in a resist layer on a silicon substrate. The total sampler size is $35 \mathrm{~mm} \times 35 \mathrm{~mm}$ and each diffraction structure occupies an area of $4 \mathrm{~mm} \times 4 \mathrm{~mm}$ separated from each other with gaps.

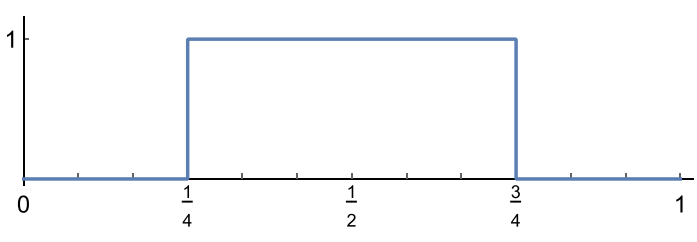

binary (2L)

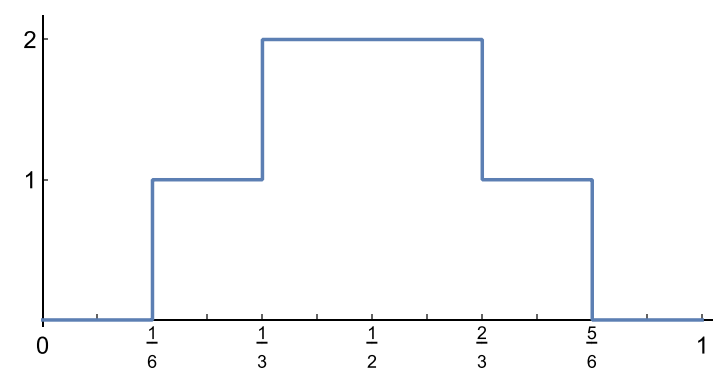

tertiary $(3 \mathrm{~L})$

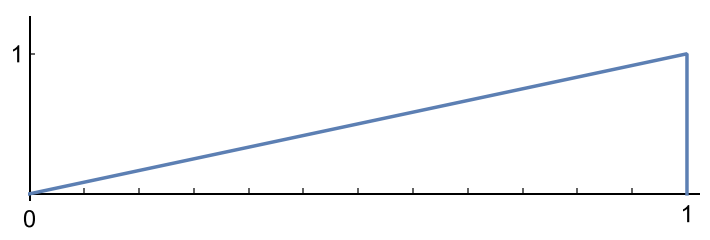

blazed (16L)

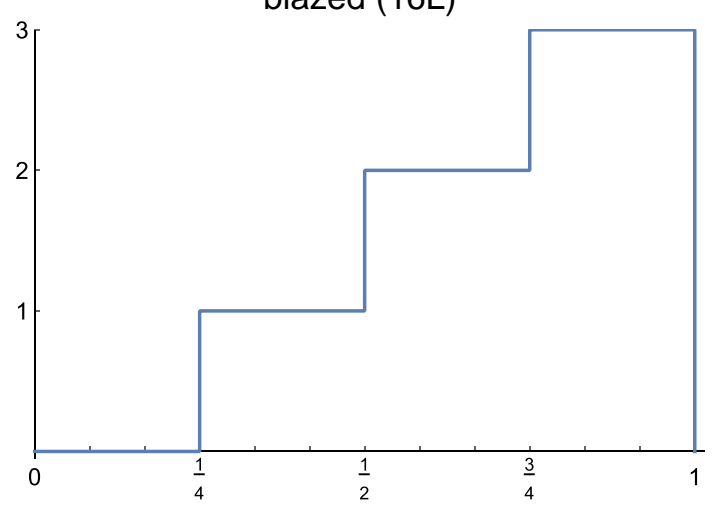

quaternary (4L)

Figure 1 Binary (2L), blazed (16L), tertiary $(3 \mathrm{~L})$ and quaternary $(4 \mathrm{~L})$ profiles used in sampler

\section{ELECTROFORMING AND EMBOSSING}

As it is common practice in manufacturing micro- and nanostructures, a master is first copied into a sturdier material before it is mass replicated. These materials are often required to endure higher pressures, higher temperatures and to be good heat conductors which is fulfilled with variety of metals. Such an ideal material is nickel that is widely used for shims for embossing processes as it is also the case of our contribution. Main steps used in our production processes are shown in schematic Figure 2. First step after successful completion of the master is a vacuum metallization. From this essential step, the non-conducting photoresist of master is covered with thin conducting layer of silver which is necessary for following electroforming. The electroforming generally produces metal layers from assembling molecules from an electrolyte on conducting surfaces. Since a sturdy sheet is required for further applications, thickness of created nickel layers ranges from tenths of microns to hundreds of microns. The galvanic process itself (electroforming) actually comprises successive production of three nickel sheets. The first one is a galvanic replica of the master (a negative), the second 
replicates the first (a positive), and the third nickel sheet, being the replica of the second, is the final galvanic replica of the master (the negative again), which is a shim used for embossing (to create the positive imprint again). The structures were embossed in polymer foils (metallized or coated with dielectrics of high refractive index and with special layers facilitating transfer of relief patterns from the shim). The embossing process is performed on a laboratory-made roll-to-roll system that is designed for a narrow (around $5 \mathrm{~cm}$ ) foil transport. A shim is clamped to a heated central roll, to which a foil is pneumatically pressed with two pressure rolls. With the system, only an embossing period of $250 \mathrm{~mm}$ is allowed which is given by used shims length $125 \mathrm{~mm}$ and transport of the foil that is set to continually emboss from the one pressure roll and in between from the second pressure roll.

The production steps were accompanied with AFM analyses of 3D reliefs and profiles of all the structures of the sampler, namely of the master, the third galvanic replica and the final pressing in a foil. The measurements of the master have to be done before the galvanic replication because the master is destroyed during it. Moreover in principle, it is not possible to make scanning electron microscope (SEM) images to analyze crosssectional cuts of the structures of the master because it is subsequently used in the galvanic replication.

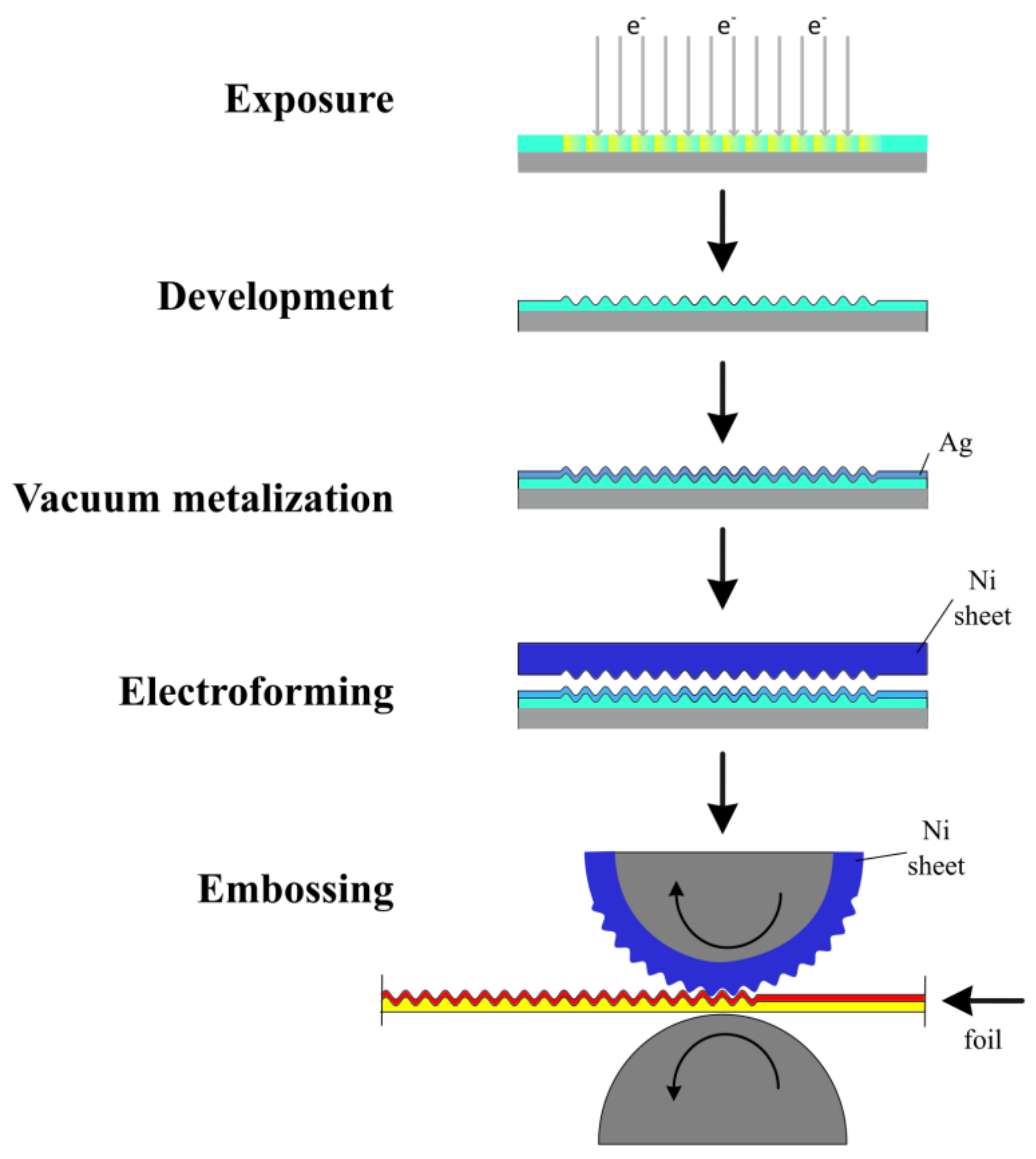

Figure 2 Realization steps of the sampler of diffraction structures

\section{RESULTS AND DISCUSSIONS}

Deviations of the profile of a final embossed structure from that of the master are almost ubiquitous in practice and can generally consists in a loss of modulation depth, a change of the period and a deformation of its shape. Throughout the structures on the sampler, these deviations were carefully studied and analyzed according to previous AFM measurements. Results of the analyses of the studied structures in a form of their profiles for a 
few periods are presented in Figure 3. Although one example of the profile is shown for each structure type from each part of the sampler, the following evaluations of the fidelity of profiles is based on the results of all the structures from the sampler.

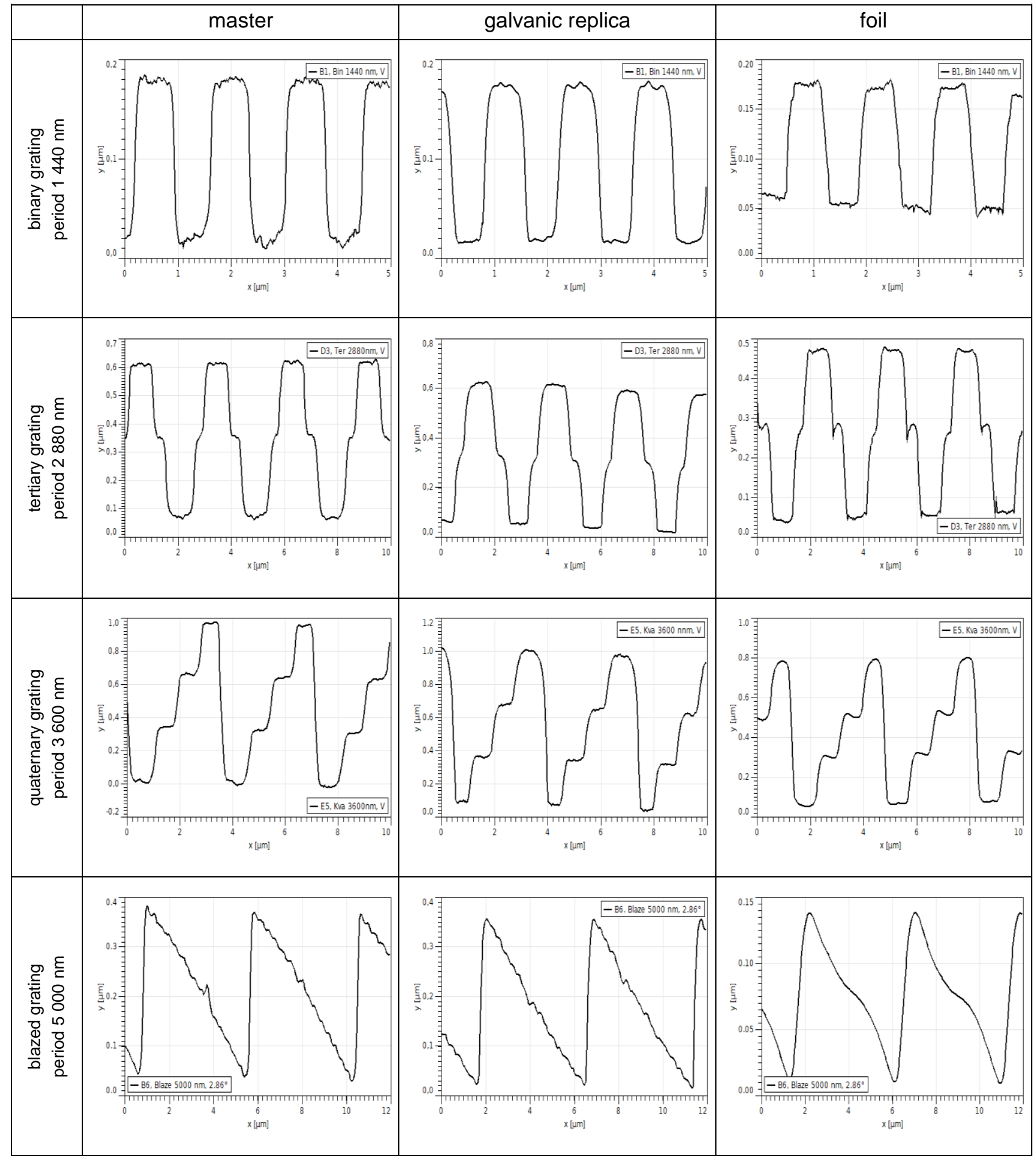

Figure 3 Profiles of the selected binary (2L), tertiary (3L), quaternary (4L) and blazed (16L) gratings from master, galvanic replica and foil

The electroforming process seems to cause some deformations of the profile shape including a certain decrease of the modulation depth and a very small change of a grating period may occur. As can be seen from 
the AFM images of grating profiles that were measured (see Figure 3) on the sampler's master and its Ni-replica grating structures, electroforming causes certain deformations of corresponding profile shapes. Especially, the tertiary and quaternary ones are affected. Generally, the more significant the deformations are, the shorter the grating period and this phenomenon does not seem to be essential for periods about approximately $2 \mu \mathrm{m}$ or larger. There is a drop in modulation depth of the tertiary gratings and quaternary gratings for shortest periods $(720 \mathrm{~nm})$ of approximately $50 \%$. The absolute depths of other grating structures of $150 \mathrm{~nm}-1,000 \mathrm{~nm}$ roughly matches the assumptions.

On the other hand, deformations of the embossed profiles is more significant compared to the corresponding master ones for large periods (above $2 \mu \mathrm{m}$ ), which could be seen best in the case of the binary shape. An explanation of apexes and valleys occurring at the bottom and top parts of the profile might be sought in different technological conditions at the edges and central part of both cavities and protrusions of the Ni-replica [4-7] but more detailed inquiries into this subject would be beyond the scope of this contribution. In the case of tertiary and quaternary profiles, deformations is rather difficult to describe but again it appears more evident with the increase of the grating period. There is a drop in modulation depth between Ni-replica and foil of approximately $20 \%-25 \%$. The more pronounced drop in modulation depth occurred for the shortest periods $(720 \mathrm{~nm})$ of the tertiary gratings and quaternary gratings and for largest periods $(4,320 \mathrm{~nm})$ for all the binary, tertiary and quaternary gratings.

Deformations of the blazed profiles, which were generated as 16 -level stairs-like structures for the periods in the range 2,000 $\mathrm{nm}-20,000 \mathrm{~nm}$, deserves a special attention. There is a $30 \%-40 \%$ drop of the modulation depth (compared to the master) in the whole period range. For periods larger than a certain limit, the embossed profiles are much less inclined in its central part than its master or shim counterpart while the profile inclination increases towards both the profile minimum and maximum. The limit is affected by modulation depths and approximately, it is lower for higher modulations. While deformations becomes significant for the periods above $5,000 \mathrm{~nm}$ for the design depth of about $250 \mathrm{~nm}-300 \mathrm{~nm}$, with a line of division around the period of 5,000 nm (see the profile in Figure 3 at the bottom right), a deeper profile (with the design depth around 1,000 nm) is much more deformed for the same grating period and it is relatively undeformed though shallower, for the grating period of $2,000 \mathrm{~nm}$.

\section{CONCLUSIONS}

The sampler of 36 mesh diffraction structures - binary, tertiary, quaternary and blazed gratings - was realized, measured and analyzed. The electron-beam writing technology was used to create the master, followed by the three steps of galvanic replication and embossing in polymer foils on a roll-to-roll system.

For the sake of this short contribution, we used the most straightforward quantification of grating profiles fidelity of the copies in comparison to their master. This means only those deformations that are clearly recognizable by an eye from measured profiles (Figure 3) were considered significant and discussed. A more rigorous approach is certainly possible (e.g. employment of convolution operators) but its scope would be a matter of standalone analyses.

The first reproduction method, electroforming of nickel shims, does not cause significant deviations of shapes or modulation depths in the whole studied range of periods of the binary profile $(720 \mathrm{~nm}$ to $4,320 \mathrm{~nm})$, of the blazed profile $(2,000 \mathrm{~nm}$ to $20,000 \mathrm{~nm})$. The same applies to deviations of the tertiary profile in the range from about $1,400 \mathrm{~nm}$ to $4,320 \mathrm{~nm}$, of the quaternary profile in the range from about $2,160 \mathrm{~nm}$ to $4,320 \mathrm{~nm}$.

The second reproduction method, embossing, causes significant deformations not only for the smallest period but in a much more significant manner for the longest ones as well. The latter can be seen best for the binary and blazed profile but deformations occur for the tertiary and quaternary profiles too. 
Overall, faithful reproduction of the master engages two important aspects. One is limited "resolution" of both the electroforming and the embossing that plays a role mainly with profiles of the smallest periods. The other is related to rheological properties of liquefied foil materials during embossing and thus it is appearing more significantly for the larger periods. However, deviation of the modulation depth of the embossed profiles alone may not completely follow faithful reproduction with any mentioned aspect.

\section{ACKNOWLEDGEMENTS}

The research was partially supported by the Ministry of the Interior of the Czech Republic (project No. VI20192022147), and by CAS (RVO:68081731).

\section{REFERENCES}

[1] RENESSE, R. L. van. Optical Document Security. 3rd edition. Boston/London: Artech House, 2005, pp. 386. ISBN 1-58053-258-6.

[2] MELUZÍN, P. et al. Some Other Gratings: Benchmarks for Large-Area E-Beam Nanopatterning. In: NANOCON 2014: 6th Int'l Conference Proceedings. Ostrava: TANGER, 2014, pp. 246-251.

[3] KOLAŘíK, V. et al., Structural Color of Metallic Surfaces. In: METAL 2014: 23rd International Conference on Metallurgy and Materials. Ostrava: TANGER, 2014, pp. 962-967.

[4] SCHIFT, H. et al., Pattern formation in hot embossing of thin polymer films. Nanotechnology. 2001, vol. 12, pp. 173-177.

[5] HEYDEMAN, L. J. et al. Flow behavior of thin polymer films used for hot embossing lithography. Microelectronic Engineering 2000, vol. 54, no. 3-4.

[6] TORRES, C. M. S. Alternative Lithography. US: Springer, 2003.

[7] PENG, L. et al. Micro hot embossing of thermoplastic polymers: a review. J. Micromech. Microeng. [online]. 2014, vol. 24, pp. 013001-013023. Available from: https://doi.org/10.1088/0960-1317/24/1/013001. 